\title{
Incubation period of coronary heart disease
}

\author{
GEOFFREY ROSE
}

\begin{abstract}
Serum cholesterol concentrations and blood pressure were measured during 1958-64 among men aged 40-59 who took part in the Seven Countries Study. In the present study these measurements were related to the national mortality from coronary heart disease in the periods 1959-61, 1964-6, 1969-71, and 1974-6. The correlations increased with time $(r=+0.86,0.90,0.93$, and 0.96 respectively for serum cholesterol concentration and $\mathbf{r}=+0 \cdot 48,0.56,0.57$, and 0.64 for systolic blood pressure), suggesting that the "incubation period" between exposure to major coronary risk factors and the maximum effects on mortality may be 10 years or more.
\end{abstract}

\section{Introduction}

The interval that separates exposure to a cause from the onset of illness (incubation period) is difficult to study in diseases that result from chronic exposure, such as cancers or atherosclerosis. The results of animal studies may not apply in man. Intervention studies may indicate the interval from reduction of exposure to abatement of risk, but this is a different question-for example, it might take years of exposure to hyperlipidaemia or hypertension for a critical level of atherosclerosis to be reached, even though intervention trials suggest a swiftly appearing benefit. ${ }^{12}$

I have examined some indirect evidence on the interval between exposure of populations to certain levels of risk factors and the maximum correlation with subsequent mortality from coronary heart disease.

\section{Methods}

During 1958-64 Keys $e t$ al $^{3}$ recorded levels of coronary risk factors in 14 population samples of men aged 40-59 drawn from seven countries-namely, Finland, Greece, Italy, Japan, the Netherlands, USA, and Yugoslavia. They then related these initial characteristics to the five-year and 10-year incidences of coronary heart disease in the 14 cohorts. For the present analysis results from this Seven Countries Study for separate population samples from the same country were combined, yielding "best estimates" of the serum cholesterol concentrations and blood pressure prevailing at that time among men aged 40-59 in the seven countries. These were then related to the national mortality from coronary heart disease ${ }^{4}$ in the same age cohorts at five-year intervals over the next 15 years. Each quinquennial estimate was based on the average of mortality rates over three separate years, age-adjusted by averaging the age-specific rates (expressed in five-year classes).

Mortality rates naturally increase with time as the cohorts grow older, and as a result the absolute differences in mortality between countries also increase. To compensate for this irrelevant effect rates were plotted on a logarithmic scale.

\section{Results}

Figure 1 shows the relation of initial mean serum cholesterol concentrations to concurrent and subsequent mortality from coronary

\footnotetext{
Department of Medical Statistics and Epidemiology, London Schoo of Hygiene and Tropical Medicine, London WC1E 7HT

GEOFFREY ROSE, DM, FRCP, professor of epidemiology
}

heart disease in the seven countries. The correlation coefficient ( $r$ ) was already +0.86 for the initial rates; it increased steadily over successive five-year intervals ( 0.90 after five years, 0.93 after 10 years, and 0.96 after 15 years). Correlation with the logarithms of rates gave similar results $(r=+0.87,0.91,0.98$, and 0.97 respectively). Over the 15 years there was little change in the slopes of the regressions of the logarithm of rates on initial cholesterol concentrations - that is to say, the magnitude of the predictive effect stayed about the same-but the accuracy (measured by the closeness of correlation about the regression line) increased with time. In the initial correlation Greece showed an anomalously low mortality whereas the USA anomalously had a higher rate than Finland; but after 10 and 15 years the national rankings for cholesterol and mortality were identical.

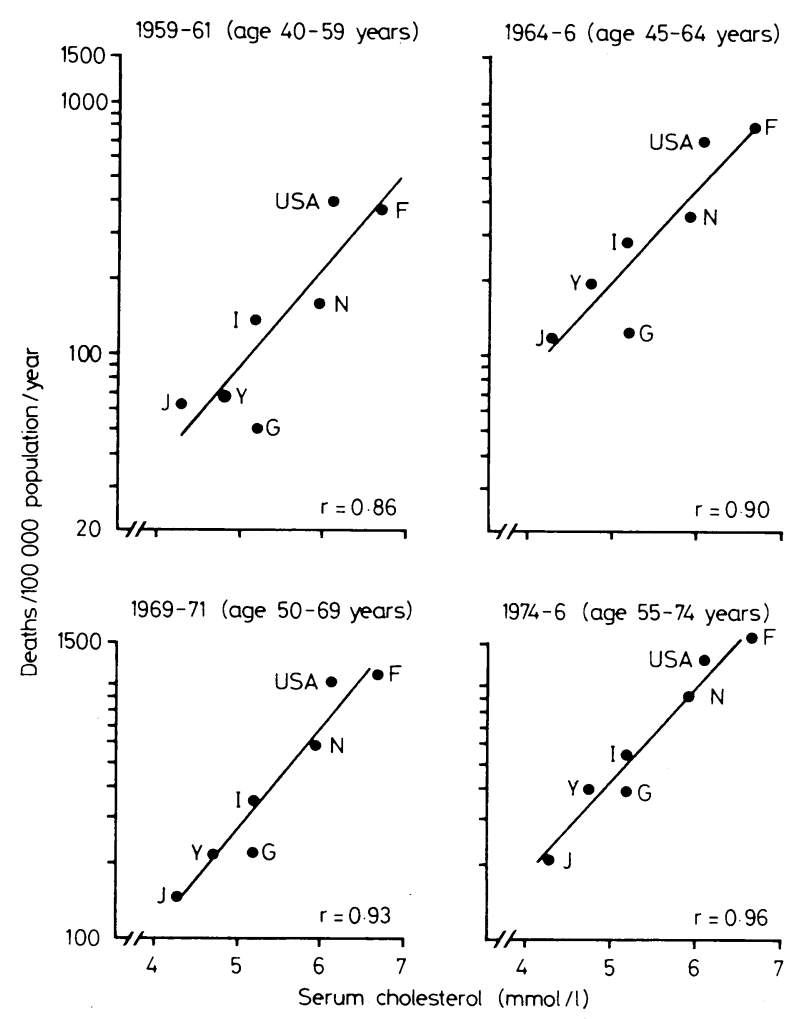

FIG 1 -Correlations and fitted linear regressions between serum cholestero concentrations during 1958-64 and age-adjusted mortality from coronary heart disease among corresponding cohorts of men, initially aged 40-59 years, in 1959-61, 1964-6, 1969-71, and 1974-6.

$\mathrm{F}=$ Finland; $\mathrm{I}=\mathrm{Italy} ; \mathrm{N}=$ the Netherlands; $\mathrm{J}=$ Japan; $\mathrm{Y}=$ Yugoslavia $\mathrm{G}=\mathrm{Greece}$.

Conversion: SI totraditional units-Cholesterol: $1 \mathrm{mmol} / 1 \approx 38.7 \mathrm{mg} / 100 \mathrm{ml}$.

Figure 2 shows the corresponding data for systolic blood pressure. The correlations were lower but showed the same tendency to increase with time $(\mathrm{r}=+0.48,0.56,0.57$, and 0.64 with the simple rates, and $+0.64,0.68,0.71$, and 0.72 with the logarithms of the rates). Figure 3 shows the data for diastolic blood pressure. The predictions were a little weaker than those for systolic pressure but again improved with time $(r=+0.34,0.41,0.46$, and 0.53 with the simple rates, and $+0.51,0.58,0.66$, and 0.70 with the logarithms of the rates). Given that the correlation of cholesterol concentration with mortality from coronary heart disease after 15 years was almost perfect $(r=0.96)$, it is not surprising that in a multiple correlation test the addition of systolic or diastolic pressure gives only a small and non-significant improvement.

The possibility must be considered that the apparent improvement 
in prediction with increasing time might simply reflect some statistical effect of an aging population. Calculations were therefore repeated, relating initial cholesterol concentration to mortality from coronary heart disease in men aged 55-64 in each of the four time periods. Again, the predictions tended to improve with time $(r=+0.86,0.91$, $0 \cdot 94$, and $0 \cdot 95$ ).

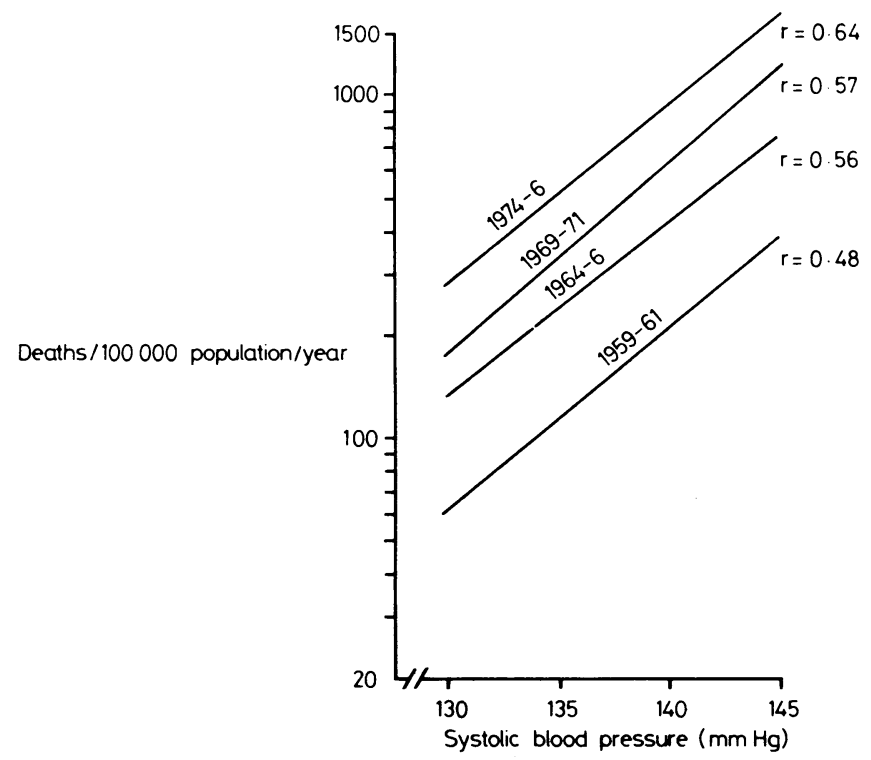

FIG 2-Correlations and fitted linear regressions between systolic blood pressure during 1958-64 and age-adjusted mortality from coronary heart disease among corresponding cohorts of men.

During the course of this analysis it was noted that cholesterol concentrations were also positively correlated with mortality from "all causes" $(r=+0.41,0.61,0.59$, and 0.69 for the four time periods, using logarithms of rates) and mortality from "all cancers" ( $r=+0.63$, $0.69,0.75$, and 0.69 respectively). There were also strong but inverse correlations with deaths ascribed to senility and ill-defined causes.

\section{Discussion}

The serum cholesterol concentrations of the local (mainly rural) population samples in the Seven Countries Study appear to have truly reflected the national differences (though not necessarily the national averages), since they correlate so remarkably well with the national differences in mortality. The high correlations also vindicate the use of national mortality rates, since errors in the data would tend to weaken the correlation estimates rather than create false-positive associations. Laboratory biases are excluded by using a central laboratory, and a false-positive correlation would seem possible only if there had been a tendency in countries with a high incidence of coronary heart disease to select population samples with unrepresentatively high cholesterol concentrations or vice versa in countries with a low incidence of the disease. Nevertheless, the strong inverse correlation of cholesterol concentration with senility and ill-defined causes of death indicates that this is also a broad marker of medicosocial conditions and not only of dietary fat.

The results support and extend the conclusion from the Seven Countries Study that differences in serum cholesterol concentration account for most of the differences in mortality from coronary heart disease between these countries. Differences in blood pressure might also contribute, but any such effect is overshadowed by the strength of the cholesterol prediction; furthermore, the comparison might be confused by measurement biases.

All the correlations (for cholesterol concentration and both systolic and diastolic blood pressures) grew steadily stronger with time, and the cholesterol concentrations in the late 1950s gave an almost perfect prediction of the international differences in mortality from coronary heart disease in the mid-1970s. With data from only seven countries it would be unwise to put too much reliance on the time trend, which was consistent with a delay of 10 years or more between exposure of a population to given concentrations of cholesterol or blood pressure and the maximum effect on mortality from coronary heart disease; the decline in American rates in the 1970s brought them into line

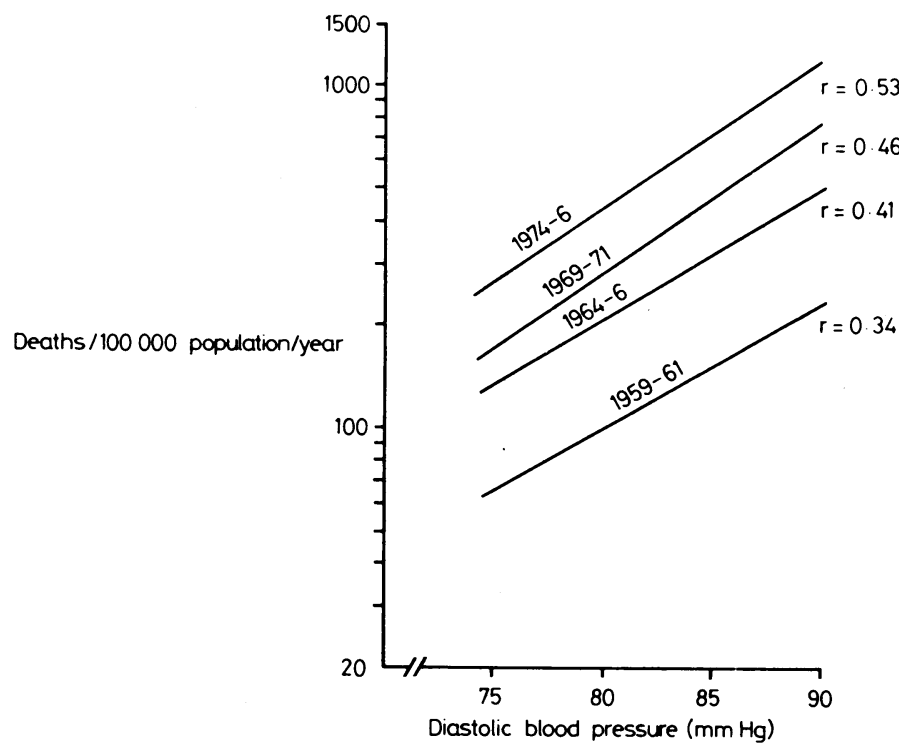

FIG 3-Correlations and fitted linear regressions between diastolic blood pressure during 1958-64 and age-adjusted mortality from coronary heart disease among corresponding cohorts of men.

with what would have been predicted by the cholesterol concentration more than a decade earlier. The situation is, however, complicated by continuing changes in the levels of these and other coronary risk factors. This makes it all the more surprising that a single examination can permit such accurate long-term predictions.

Longitudinal surveys such as the Framingham study ${ }^{5}$ have found that in individuals the predictive ability of the serum cholesterol concentration is much less in older than in younger people, which seems to be at variance with the present findings. Possibly the determination of individual risk has a different basis from the determination of rates in populations. Some clarification might come from comparing short-term and longterm predictive power within the same individuals.

The results do not support the view that low mean concentrations of cholesterol imply a higher national risk of cancer: the trend is in fact rather strongly in the reverse direction.

Data extraction and computing were undertaken by Miss Linda Colwell.

\section{References}

${ }^{1}$ Hjermann I, Byre VK, Holme I, Leren P. Effect of diet and smoking intervention on the incidence of coronary heart disease. A randomised trial in healthy men. The Oslo Study. Lancet 1981 ;ii:1303-10.

${ }^{2}$ Hypertension Detection and Follow-up Program Cooperative Group. Five-year findings of the hypertension detection and follow-up program. FA.MA 1979;242:2562-71.

${ }^{3}$ Keys A, and other authors. Coronary heart disease in seven countries. Circulation 1970;41-42,suppl:1-211.

4 WHO. World health statistics annuals, 1962-76. Geneva: WHO, 1965-81.

5 Kannel WB, Castelli WP, Gordon T, McNamara PM. Serum cholesterol, lipoproteins, and the risk of coronary heart disease. Ann Intern Med 1971;74:1-12.

(Accepted 2 April 1982) 